\title{
Transcriptome sequencing in an ecologically important tree species: assembly, annotation, and marker discovery
}

\author{
Thomas L Parchman ${ }^{1 *}$, Katherine S Geist ${ }^{2}$, Johan A Grahnen ${ }^{3}$, Craig W Benkman ${ }^{4}$, C Alex Buerkle ${ }^{1}$
}

\begin{abstract}
Background: Massively parallel sequencing of cDNA is now an efficient route for generating enormous sequence collections that represent expressed genes. This approach provides a valuable starting point for characterizing functional genetic variation in non-model organisms, especially where whole genome sequencing efforts are currently cost and time prohibitive. The large and complex genomes of pines (Pinus spp.) have hindered the development of genomic resources, despite the ecological and economical importance of the group. While most genomic studies have focused on a single species ( $P$. taeda), genomic level resources for other pines are insufficiently developed to facilitate ecological genomic research. Lodgepole pine (P. contorta) is an ecologically important foundation species of montane forest ecosystems and exhibits substantial adaptive variation across its range in western North America. Here we describe a sequencing study of expressed genes from $P$. contorta, including their assembly and annotation, and their potential for molecular marker development to support population and association genetic studies.
\end{abstract}

Results: We obtained 586,732 sequencing reads from a 454 GS XLR70 Titanium pyrosequencer (mean length: 306 base pairs). A combination of reference-based and de novo assemblies yielded 63,657 contigs, with 239,793 reads remaining as singletons. Based on sequence similarity with known proteins, these sequences represent approximately 17,000 unique genes, many of which are well covered by contig sequences. This sequence collection also included a surprisingly large number of retrotransposon sequences, suggesting that they are highly transcriptionally active in the tissues we sampled. We located and characterized thousands of simple sequence repeats and single nucleotide polymorphisms as potential molecular markers in our assembled and annotated sequences. High quality PCR primers were designed for a substantial number of the SSR loci, and a large number of these were amplified successfully in initial screening.

Conclusions: This sequence collection represents a major genomic resource for $P$. contorta, and the large number of genetic markers characterized should contribute to future research in this and other pines. Our results illustrate the utility of next generation sequencing as a basis for marker development and population genomics in nonmodel species.

\section{Background}

Large numbers of molecular markers and sequence data from across the genome are playing an increasingly important role in population genomic studies of finescale genetic variation and the genetic basis of traits [1]. Nevertheless, we lack genomic resources for most nonmodel organisms and whole genome sequencing is still

\footnotetext{
* Correspondence: tparchma@uwyo.edu

'Department of Botany, University of Wyoming, Laramie, WY 82071, USA
}

largely impractical for most eukaryotes. Transcriptome, or Expressed Sequence Tag (EST), sequencing is an efficient means to generate functional genomic level data for non-model organisms or those with genome characteristics prohibitive to whole genome sequencing. EST sequencing is an attractive alternative to whole genome sequencing because the majority of most eukaryotic genomes is non-coding DNA, and EST sequences lack introns and intragenic regions that render analysis and interpretation of data more difficult [2]. ESTs thus have 
a high functional information content, and often correspond to genes with known or predicted functions [2,3]. Large collections of EST sequences have proven invaluable for gene annotation and discovery $[2,4]$, comparative genomics [5], development of molecular markers $[6,7]$, and for population genomic studies of genetic variation associated with adaptive traits [8]. Nonetheless, until recently, traditional laboratory methods for the development of EST resources have required costly and time consuming approaches involving cloning, cDNA library construction, and many labor intensive Sanger sequencing runs [2].

Massively parallel sequencing technologies, such as 454 pyrosequencing, remove many time consuming steps involved in Sanger sequencing of ESTs and have facilitated transcriptome sequencing at a fraction of the time and cost previously required [5,9-11]. At present, a single run on a 454 GS XLR70 Titanium pyrosequencer can produce more than $10^{6}$ sequences averaging greater than 300 base pairs (bp) in length. The de novo assembly of the large numbers of short reads produced from this and similar technologies is a significant challenge for whole genome sequencing of large and complex genomes. In contrast, for transcriptome sequencing, de novo assembly is facilitated by the possibility of increased coverage depth (number of reads per nucleotide in the template) for the much smaller number of nucleotides in the transcriptome than in the whole genome [4]. In addition, the reduced amount of repetitive DNA found in genes compared to non-coding regions ameliorates one of the principal obstacles to de novo assembly of short reads [12]. Whereas most applications of parallel sequencing of ESTs have involved model organisms with draft genomes available to aid in assembly $[4,13,14]$, recent studies have demonstrated highly successful de novo assemblies of 454 EST data for organisms with no prior genomic resources $[5,7,15,16]$. The generation of such largescale sequence data will enable functional analyses that were previously limited to model organisms and their rapid application in ecologically important taxa [17]. Here, we utilize pyrosequencing of cDNA to characterize the transcriptome of lodgepole pine (Pinus contorta) and to develop genomic resources to support further research in this and other pines.

$P$. contorta is an ecologically and economically important tree that is widespread in the mountainous regions of western North America [18]. It is a fire-adapted species that mediates regeneration after disturbance, has a major impact on forest structure and ecology, and is a foundation species of many montane forest ecosystems. It is one of the most variable pines, and grows in a variety of conditions ranging from low elevations to timberline [19] where it has experienced and evolved in response to diverse selection pressures including that from variation in seed predator communities [20-23] and fire regime $[24,25]$. The current mountain pine bark beetle (Dendroctonus ponderosae) epidemic is causing unprecedented mortality of $P$. contorta throughout the Rocky Mountains [26], which is likely to cause rapid and massive changes in community structure and ecosystem processes. Consequently, a greater understanding of fine-scale population genetic variation and the genetic control of traits important to these forests would be beneficial and timely.

Although a large number of EST sequences for loblolly pine (P. taeda) exist in public databases (e.g., NCBI), far fewer resources exist for $P$. contorta (1 EST prior to 2010, as of January 2010 ca. 40,000 ESTs) and other pines, despite the importance of the genus. This paucity exists in part because pines have enormous genomes (10,000-40,000 mega-base pairs vs. $115 \mathrm{Mbp}$ in Arabidopsis thaliana) with large amounts of repetitive DNA $[27,28]$, making whole genome sequencing projects difficult or impractical. The construction of large EST collections is thus the most promising approach for providing functional genomic level information in pines [29]. Whereas other labs are currently generating $P$. contorta ESTs using Sanger sequencing (K. Ritland and J. Boehlman, pers. comm.), additional sequencing effort is needed to increase genomic level resources. The development of genomic resources for $P$. contorta should facilitate basic and applied research on the genetics and evolutionary ecology of this species and its role in maintaining forest health and ecosystem function $[29,30]$. In addition, EST collections for P. contorta will contribute to the development of molecular markers for other pines and facilitate comparative genomics and the study of adaptive variation across the genus.

Here we describe 454 pyrosequencing of $P$. contorta cDNA and assess the utility of this approach for transcriptome characterization and marker discovery in a species with a large and complex genome. Normalized cDNA collections from multiple tissues and individuals were used to sample large numbers of expressed genes and to detect simple sequence repeats (SSRs) and single nucleotide polymorphisms (SNPs). We first describe the assembly and functional annotation of EST sequences, and the level of transcriptome coverage provided by our sequence data. Second, we discuss the detection and characterization of a surprisingly large number of sequences representing retrotransposons. Finally, we utilize our assembled sequence data for the development of a variety of gene-based markers for population genomic studies, including SSRs occurring within regions that are conserved with another pine species, and SNPs occurring in regions with many reads and deep coverage. We designed high quality PCR primers for a large 
number of the SSRs we characterized, providing an immediately available resource of genetic markers for pines. Along with other recent studies $[5,7,15,16]$, our results demonstrate the utility and highlight some of the challenges of next generation transcriptome sequencing applied to non-model organisms.

\section{Results and Discussion \\ Results}

\section{4 sequencing and assembly}

We created a normalized cDNA pool based on RNA extracted from needles and developing conelets that were sampled from four individual $P$. contorta trees in the Medicine Bow National Forest in Wyoming. Pyrosequencing of this cDNA pool on a 454 GS XLR70 Titanium platform produced approximately $180 \mathrm{Mbp}$ of sequence data, in the form of 586,732 reads averaging $306 \mathrm{bp}$ in length (Fig. 1). Assuming a similar number of genes occur in $P$. contorta as in A. thaliana $(25,000)$ and a similar average gene length of 2,000 bp [31], average transcriptome coverage was estimated at $3.6 \times$. We removed a variety of reads with characteristics that could interfere with assembly prior to further analyses. After removing all reads that matched to three well- characterized conifer retrotransposons $(11,394)$ [32-34], sequences containing long simple sequence repeats $(3,681)$, and sequences not passing stringent quality screening (average quality score $<18 ; 106,761$ reads), 464,896 reads entered assemblies run in Seqman Ngen (DNAstar, Inc.).

We first evaluated how varying some of the starting parameters affected de novo assembly of reads by running 18 different assemblies with different combinations of minimum match percentage, minimum match size, and gap penalty. Predictably, the number of assembled sequences and the length of assembled contigs increased with decreasing match percentage and decreasing match size (Table 1). However, the number of contigs was largest with a match percentage of $90 \%$, suggesting that a substantial number of contigs (including paralogs) may be collapsed and joined when match percentages are dropped as low as $85 \%$ and a larger number of reads are assembled into a smaller number of contigs (Table 1). Analyses throughout this paper are based on a combination of reference-based and de novo assemblies. We first executed a reference-based assembly utilizing a set of approximately 19,000 unigenes available for $P$. taeda using a minimum match percentage of $88 \%$, a minimum

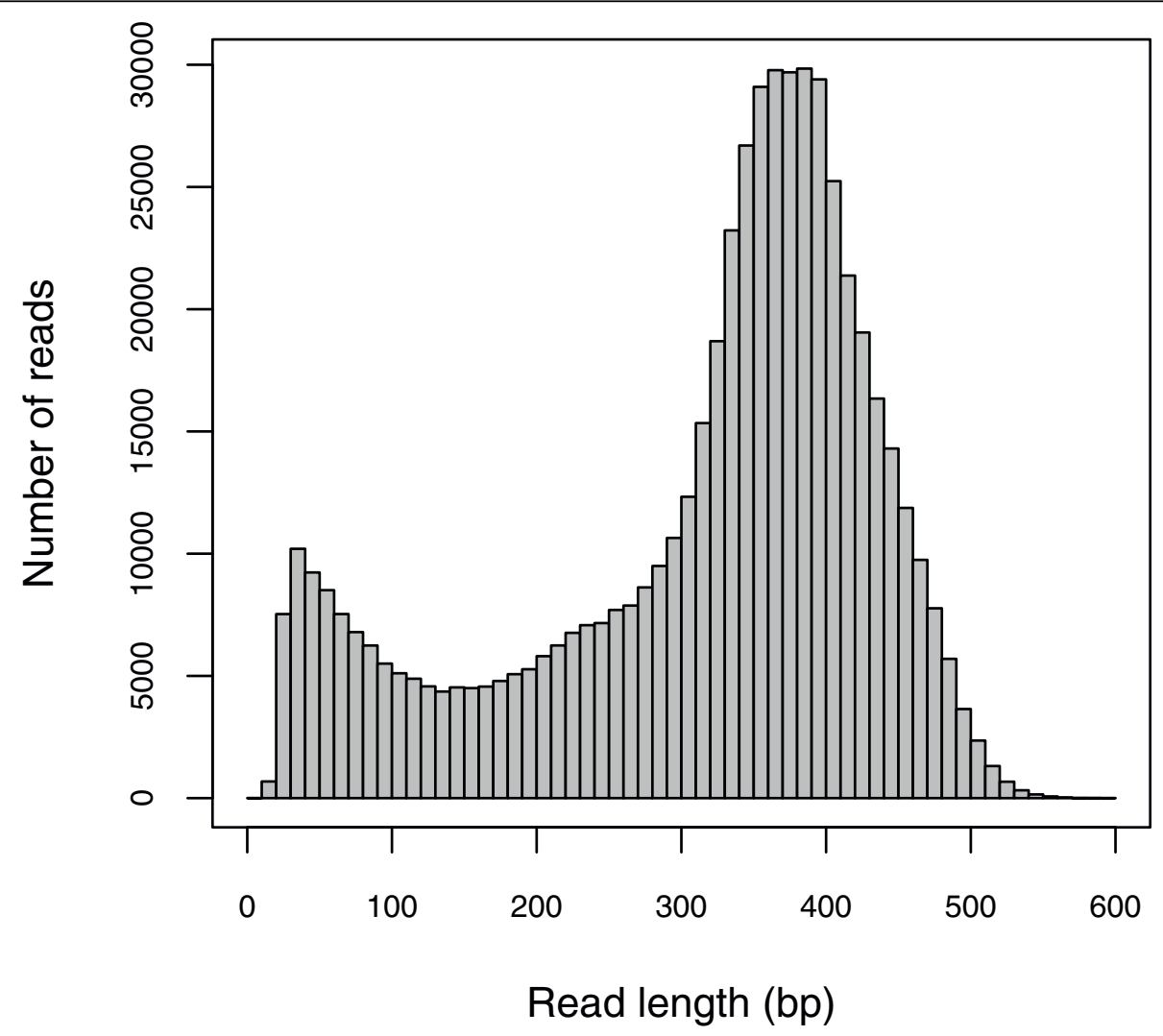

Figure 1 Frequency distribution of $\mathbf{4 5 4}$ sequencing read lengths. The frequency distribution of read lengths resulting from 454 GS XLR70 Titanium pyrosequencing. 
Table 1 Results and characteristics of assemblies run with different parameter settings

\begin{tabular}{|c|c|c|c|c|c|}
\hline \multirow[b]{3}{*}{ Match \% } & \multirow[b]{3}{*}{$\begin{array}{l}\text { Match } \\
\text { length }\end{array}$} & \multicolumn{4}{|c|}{ Gap Penalty } \\
\hline & & \multicolumn{2}{|l|}{30} & \multicolumn{2}{|l|}{50} \\
\hline & & $\begin{array}{c}\text { Number of contigs and average } \\
\text { length }\end{array}$ & $\begin{array}{l}\text { Number of assembled } \\
\text { reads }\end{array}$ & $\begin{array}{c}\text { Number of contigs and average } \\
\text { length }\end{array}$ & $\begin{array}{c}\text { Number of assembled } \\
\text { reads }\end{array}$ \\
\hline \multirow[t]{3}{*}{$85 \%$} & 19 & $50,689(537)$ & 255,711 & $51,058(533)$ & 252,299 \\
\hline & 23 & $49,621(516)$ & 248,537 & $50,039(513)$ & 245,563 \\
\hline & 25 & $48,962(507)$ & 244,120 & 49,366 (504) & 241,289 \\
\hline \multirow[t]{3}{*}{$\overline{90 \%}$} & 19 & $55,886(499)$ & 220,517 & $55,996(497)$ & 217,169 \\
\hline & 23 & $53,724(490)$ & 213,344 & $53,824(488)$ & 209,916 \\
\hline & 25 & $52,770(485)$ & 209,735 & $52,726(483)$ & 206,167 \\
\hline \multirow[t]{3}{*}{$95 \%$} & 19 & $51,289(443)$ & 156,578 & $51,908(441)$ & 154,605 \\
\hline & 23 & $46,611(441)$ & 142,428 & 46,585 (439) & 140,071 \\
\hline & 25 & $44,752(439)$ & 137,096 & $44,674(437)$ & 134,566 \\
\hline
\end{tabular}

Combination of reference-based (match \% = 88) and de novo assemblies (match \% = 93)

$\begin{array}{llll}88 \%, 93 \% & 19 & 63,687(500) & 225,517\end{array}$

Results from assemblies run in Seqman Ngen with different starting values for gap penalty, minimum match size, and mimimum match percentage. We present the number of contigs, average contig size (in parentheses), and the number of reads assembled into contigs for each different set of parameter settings.

match size of $19 \mathrm{bp}$, and a gap penalty of 30 . This assembly was executed to provide a set of contigs with sequences that are conserved between $P$. taed $a$ and $P$. contorta and to use the comparative sequence alignments to design molecular markers with high cross-species transferability. De novo assembly of the remaining reads was run with a minimum match percentage of $93 \%$, a minimum match size of $19 \mathrm{bp}$, and a gap penalty of 30. This combination of reference-based and de novo assemblies increased the number of assembled reads and the number of contigs when compared to pure de novo assembly (Table 1).

The reference-guided assembly placed 48,451 reads into 6,601 contigs (Fig. 2), which averaged $832 \mathrm{bp}$ in length and had a mean coverage depth of 2.04 (Fig. 3; skewed distribution; $25 \%$ quantile, 1.3 ; $75 \%$ quantile, 2.02). De novo assembly of reads not assembled to the $P$. taeda unigenes placed 176,652 reads into 57,086 contigs with an average length of $452 \mathrm{bp}$ and an average coverage depth of 2.1 (Fig. 3; 25\% quantile, 1.4; and 75\% quantile, 1.98). As expected, the length of contigs generally increased with the number of sequences assembled into them (Fig. 4). The combined assemblies had an average contig length of $500 \mathrm{bp}$ and contained a substantial number of large contigs (Fig. 3). 7,828 contigs were larger than $800 \mathrm{bp}$ in length, and the largest $10 \%$ were between 852 and 5,062 bp in length. 239,793 unassembled high quality reads were treated as singletons in further analyses (Fig. 2).

\section{Annotation}

To assess the coverage and quality of our assembly, we first used BLASTx [35] to align both contigs and singletons to the UniRef50 15.4 [36] and the TAIR9 Arabidopsis thaliana [37] annotated protein databases using an $E$ value threshold of $10^{-6}$. Of 63,687 contigs, 20,301 (32\%) had BLAST hits to known proteins in UniRef50 and matched 8,316 unique protein accessions (Table 2). As expected, a lower percentage of the shorter singleton reads had BLAST hits to UniRef50 proteins. Of 239,793 singleton reads, 30,836 (13\%) had blast hits to UniRef50, with matches to 10,574 unique proteins (Table 2). Smaller numbers but similar percentages of contigs and singletons had BLAST hits to the TAIR database (Table 2). The majority of the annotated sequences corresponded to known plant proteins, with $9.8 \%$ matching conifer sequences (Table 3 ). In addition, a large number of sequences $(10.4 \%)$ were most similar to fungal proteins (Table 3), likely indicating the presence of endophytic fungi in our sampled tissues. This seemingly low percentage of ESTs with BLAST hits is partially due to a high frequency of short sequences in our ESTs, although annotation of only $30-40 \%$ of sequences is common in analyses of large EST collections [5,16,38]. Longer contigs were more likely to have BLAST matches to the annotated protein databases; logistic regression indicated that EST sequence length was a significant predictor of the presence or absence of a significant BLAST match to one of the annotated protein databases (slope = 0.0058 , intercept $=-3.899, P<0.0001) .85 \%$ of our contigs and singletons over $800 \mathrm{bp}$ in length had BLAST matches, whereas only $5 \%$ of contigs and singletons shorter than 250 bp did. Nonetheless, BLAST searches identified a total of 17,321 unique protein accessions, indicating that our 454 sequencing project detected a substantial fraction of $P$. contorta genes.

Using Blast2go (v.2.3.6; [39]), we were able to assign gene ontology classes to 4,890 (31\%) of the 15,683 unique genes with BLAST matches to known proteins 


\section{Normalized cDNA}

(4 individuals, 2 tissues)

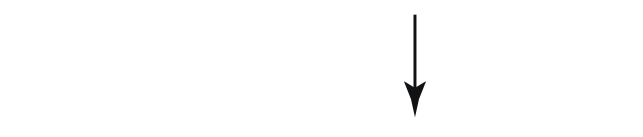

454 XLR70 Titanium Pyrosequencing

(586,732 reads averaging 306 bp)

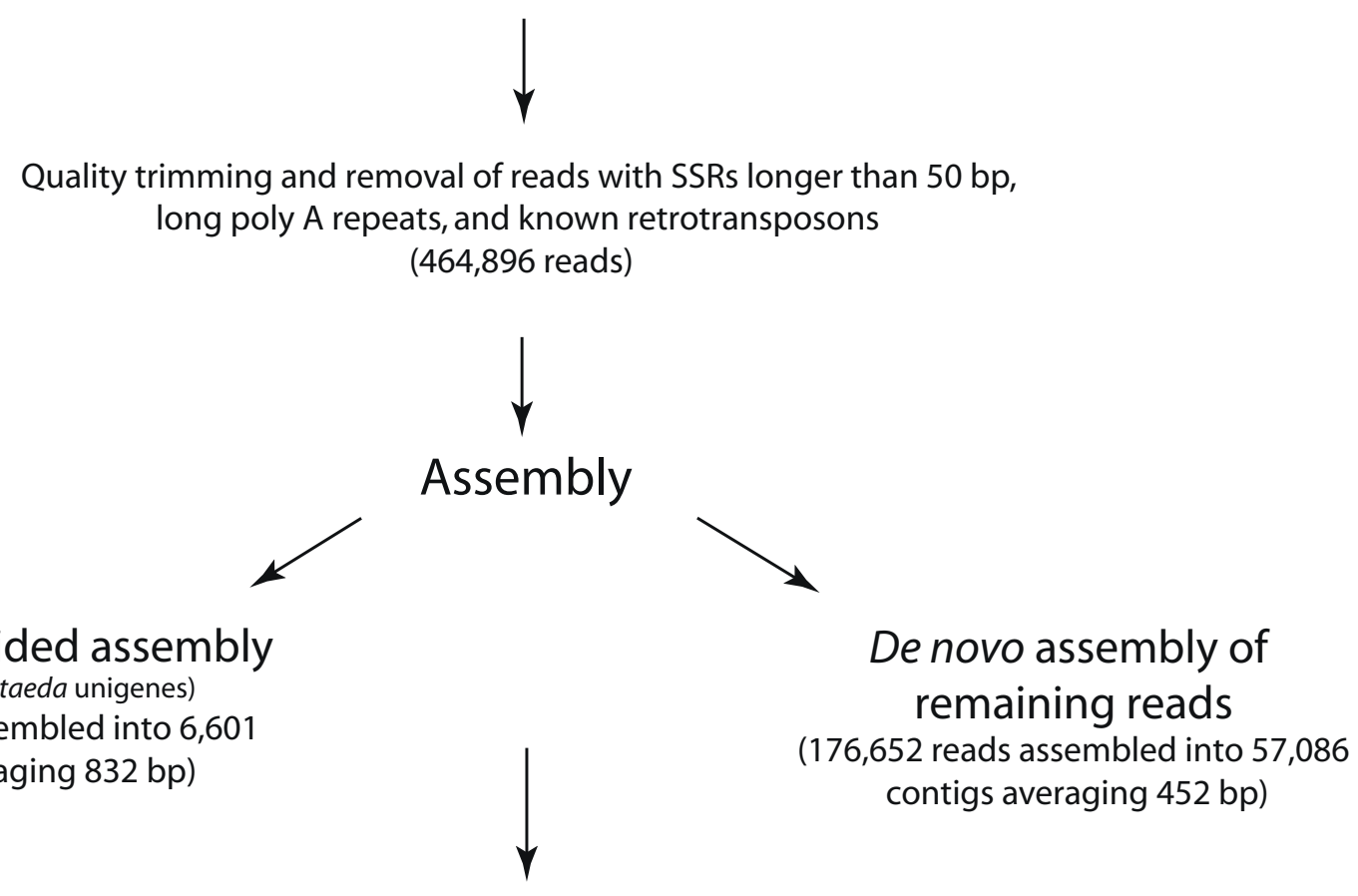

\section{3,687 contigs and 239,793 singletons}

Reference-guided assembly

(using 18,921 P.taeda unigenes)

$(48,451$ reads assembled into 6,601

contigs averaging $832 \mathrm{bp}$ )

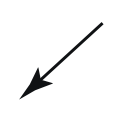

Blast \& Annotation (Uniref, TAIR)

\section{Marker Development SSRs SNPS}

Figure 2 Schematic of 454 EST analysis. The steps and sets of sequences involved in 454 EST sequencing, assembly of reads into contigs, annotation using protein databases, and genetic marker discovery and characterization.

in Uniref50. There were a total of 21,351 gene ontology terms associated with these 4,890 unique genes. Of these, assignments to the molecular function ontology made up the majority $(11,997,56.2 \%)$ followed by biological process $(5,400,25.3 \%)$ and cellular components $(3,954,18.5 \%$, Fig. 5). To compare the distribution of gene ontology annotations in our $P$. contorta 454 data to that of the $A$. thaliana genome, the unique genes from both our data and the TAIR $A$. thaliana annotated database were mapped to respective TAIR GO Slim terms using Blast2go. GO Slim terms are a specified subset of higher-level ontology categories that provide a broad profile for genome-genome comparison [40]. The percentages of annotated $P$. contorta sequences assigned to GO Slim classes generally mirrored those of $A$. thaliana genes (http://www.arabidopsis.org; Fig. 5), reflecting a similar distribution of genes in different functional categories, and further highlighting that a large diversity of $P$. contorta transcripts is represented by these sequences. 


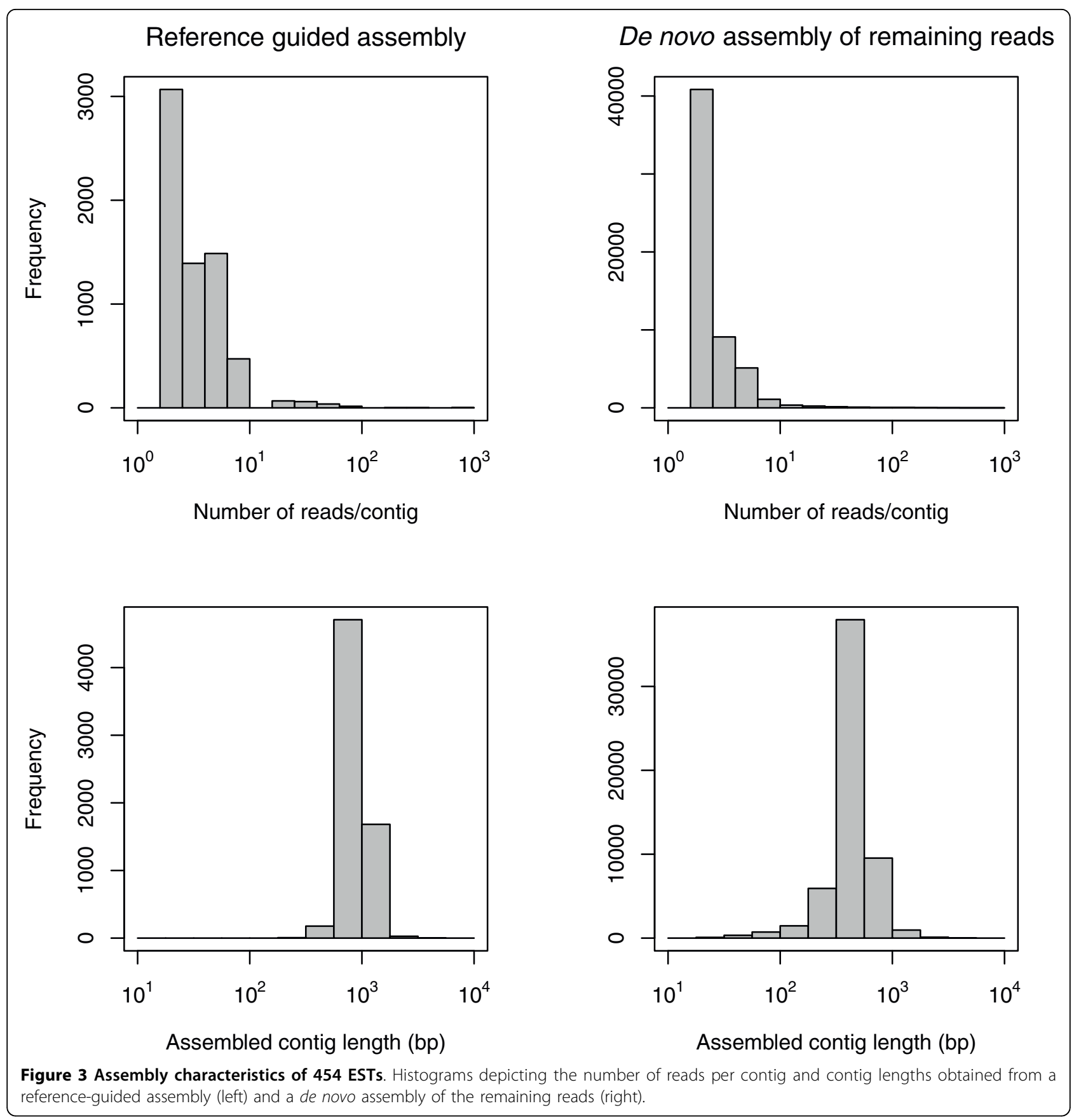

To assess the extent of transcript coverage provided by our 454 contigs and to evaluate how coverage depth affected the assembly of full length transcripts, we plotted the ratio of contig length to A. thaliana ortholog (from BLAST) length against coverage depth. Among the 454 contigs, there was a slight trend for increased coverage depth to result in higher coverage of the coding regions $\left(r^{2}=0.02, \beta=0.015, P<0.0001\right)$, although a substantial number of deeply covered contigs fail to cover complete coding regions of their A. thaliana ortholog (Fig. 6A). Values greater than 1 in Fig. 6A for the ratio of 454 contig length to the length of $A$. thaliana ortholog coding region are likely due to UTR and other non-coding regions present in our contigs, but also indicate substantial coding region coverage by many individual 454 contigs. In many cases, multiple contigs covered different regions of $A$. thaliana orthologs. Plotting the summed proportion of $A$. thaliana orthologs covered by all 454 contigs reveals that a large number of orthologs are thoroughly covered by 454 


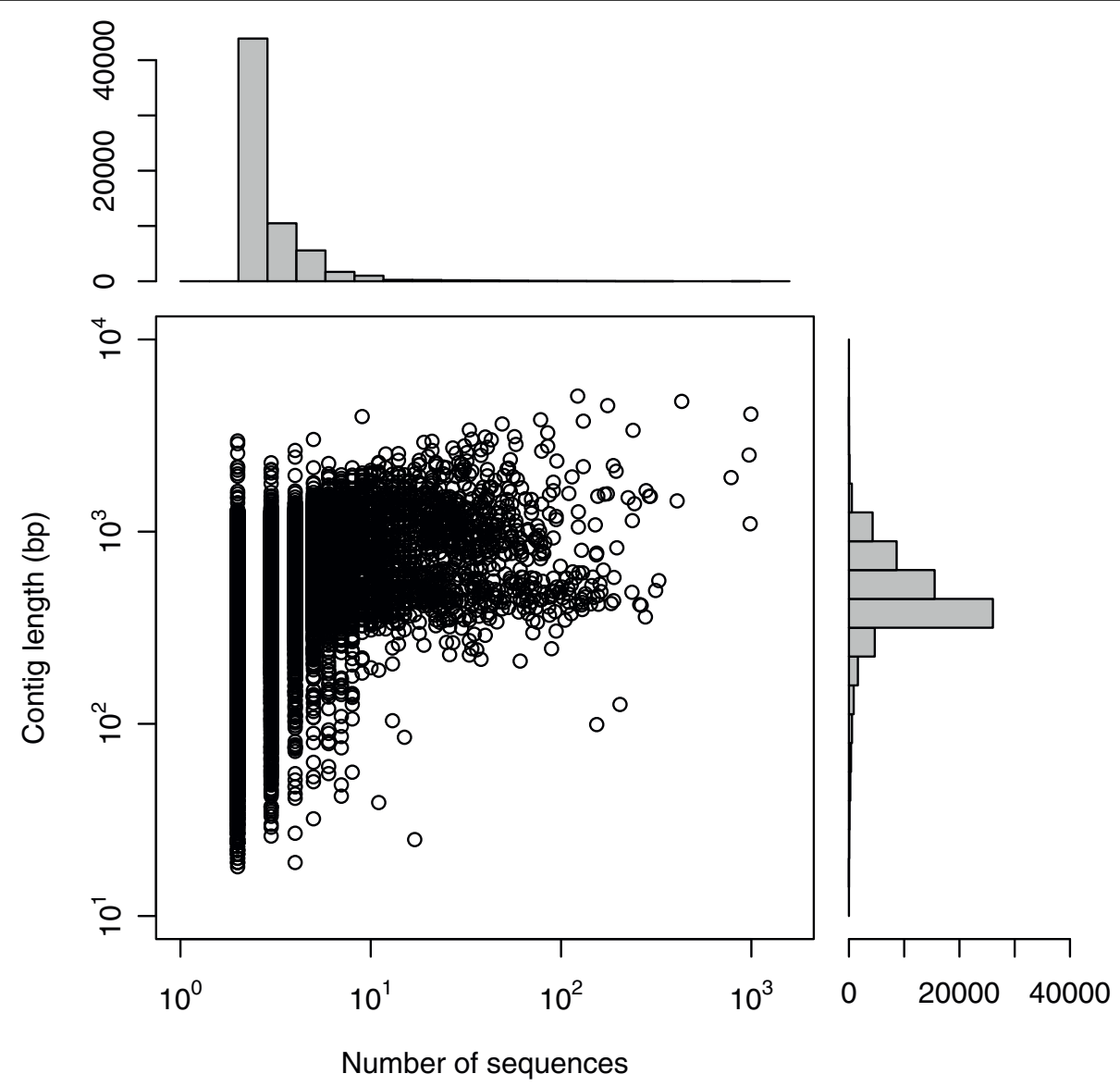

Figure 4 Contig length as a function of the number of sequences assembled into each contig. The marginal histograms depict the frequency distributions of the number of sequences assembled into contigs and the frequency distribution of contig length.

Table 2454 EST matches to annotated protein databases

\begin{tabular}{|c|c|c|c|c|c|c|}
\hline \multirow{2}{*}{ UniRef50 } & \multicolumn{2}{|c|}{ Contigs $(63,687)$} & \multicolumn{2}{|c|}{ Singletons $(239,793)$} & \multicolumn{2}{|c|}{ Combined Set $(303,480)$} \\
\hline & & & & & & \\
\hline Matches to database & 20,301 & $(32 \%)$ & 30,836 & $(13 \%)$ & 51,137 & $(17 \%)$ \\
\hline Unique matches to proteins & 8,316 & $(13 \%)$ & 10,732 & $(4 \%)$ & 15,683 & $(5 \%)$ \\
\hline \multicolumn{7}{|l|}{ TAIR9 } \\
\hline Matches to database & 11,663 & $(18 \%)$ & 12,799 & $(5 \%)$ & 24,462 & (8\%) \\
\hline Unique matches to proteins & 5,635 & $(9 \%)$ & 5,025 & $(2 \%)$ & 8,385 & (3\%) \\
\hline
\end{tabular}

Numbers and percentages of 454 ESTs in the assembled contigs, singletons, and the combined sequence set with matches to known proteins in BLASTx searches of two annotated protein databases (Uniref50, [36]; TAIR9 [37]).

contigs (Fig. 6B), although this coverage declines steeply with increasing ortholog length.

\section{Assessment of retrotransposon abundance}

BLAST searches of our 454 reads using a variety of criteria indicated an abundance of retrotransposon-like sequences in our data. Searches using queries of three known retrotransposons common in conifers (IFG7, GYMNY, and PpRT1; [32-34]) had matches to 11,394 sequences in our complete set of 454 reads. Additional searches of 17 known plant retrotransposon sequences against EST collections from ten plant taxa and our full set of raw 454 ESTs indicated that retrotransposons are present at a much higher frequency in our 454 ESTs than in other plant EST collections (Table 4). After BLAST annotation of our unique sequences, we further searched for terms identifying other proteins associated with retroelements. This resulted in an additional 3,485 unique sequences (representing a total of 13,325 reads) 
Table 3 Summary and taxonomic source of BLAST matches to 454 ESTs

\begin{tabular}{lrrrrrr}
\hline Taxonomic category & \multicolumn{2}{c}{ Contigs } & \multicolumn{2}{c}{ Singletons } & \multicolumn{2}{c}{ Combined Set } \\
\hline Conifer & 1,170 & $(14.1 \%)$ & 966 & $(9.0 \%)$ & 1,536 & $(9.8 \%)$ \\
Other plant & 4,984 & $(59.9 \%)$ & 5,191 & $(48.4 \%)$ & 8,309 & $(53.0 \%)$ \\
Insect & 363 & $(4.4 \%)$ & 1,176 & $(11.0 \%)$ & 1,413 & $(9.0 \%)$ \\
Fungi & 388 & $(4.7 \%)$ & 1,392 & $(13.0 \%)$ & 1,625 & $(10.4 \%)$ \\
Protozoa & 104 & $(11.3 \%)$ & 172 & $(1.6 \%)$ & 240 & $(1.5 \%)$ \\
Other Eukaryote & 1,208 & $(14.5 \%)$ & 1,614 & $(15.0 \%)$ & 2,260 & $(14.4 \%)$ \\
Bacteria & 82 & $(1.0 \%)$ & 199 & $(1.9 \%)$ & 270 & $(1.7 \%)$ \\
Virus & 16 & $(0.2 \%)$ & 22 & $(0.2 \%)$ & 30 & $(0.2 \%)$ \\
\hline
\end{tabular}

Number and percentages of unique best BLASTx matches of 454 EST contigs, singletons, and the combined sequence set to Uniref50 grouped by taxonomic category.

that were identified as proteins associated with retroelements. Together these three approaches indicate that as many as $36,325(6.2 \%)$ of the raw 454 reads may represent transcriptionally active retroelements.

In research on other genomes, small segments of retrotransposon sequences have been found within ESTs containing genes, which could contribute to the large number of BLAST searches matching to retrotransposon-like sequences [41]. To assess whether our sequences that aligned to Pinus retrotransposons IFG7, PpRT1, and GYMNY were located in coding regions, following [41] we assembled the corresponding sequences into 553 contigs using SeqMan Ngen. These contigs were then aligned to UniRef50 using BLASTx with an $E$ value threshold of $10^{-4}$. If pieces of retroelement sequences were in genes, we would expect these contigs to align with known proteins in UniRef50 that were non-retroelement products [41]. Of the 553 query sequences, 315 contigs had hits to UniRef50 proteins, and these sequences matched to 105 unique protein accession numbers. These accession numbers were imported into the online UniProt database search tool (http://www.uniprot.org/jobs/; [40]) and manually inspected to assess whether the associated protein products belonged to retroelements or not. Of the 105 unique proteins, 19 proteins (18.1\%) were not retroelement protein products determined by either protein

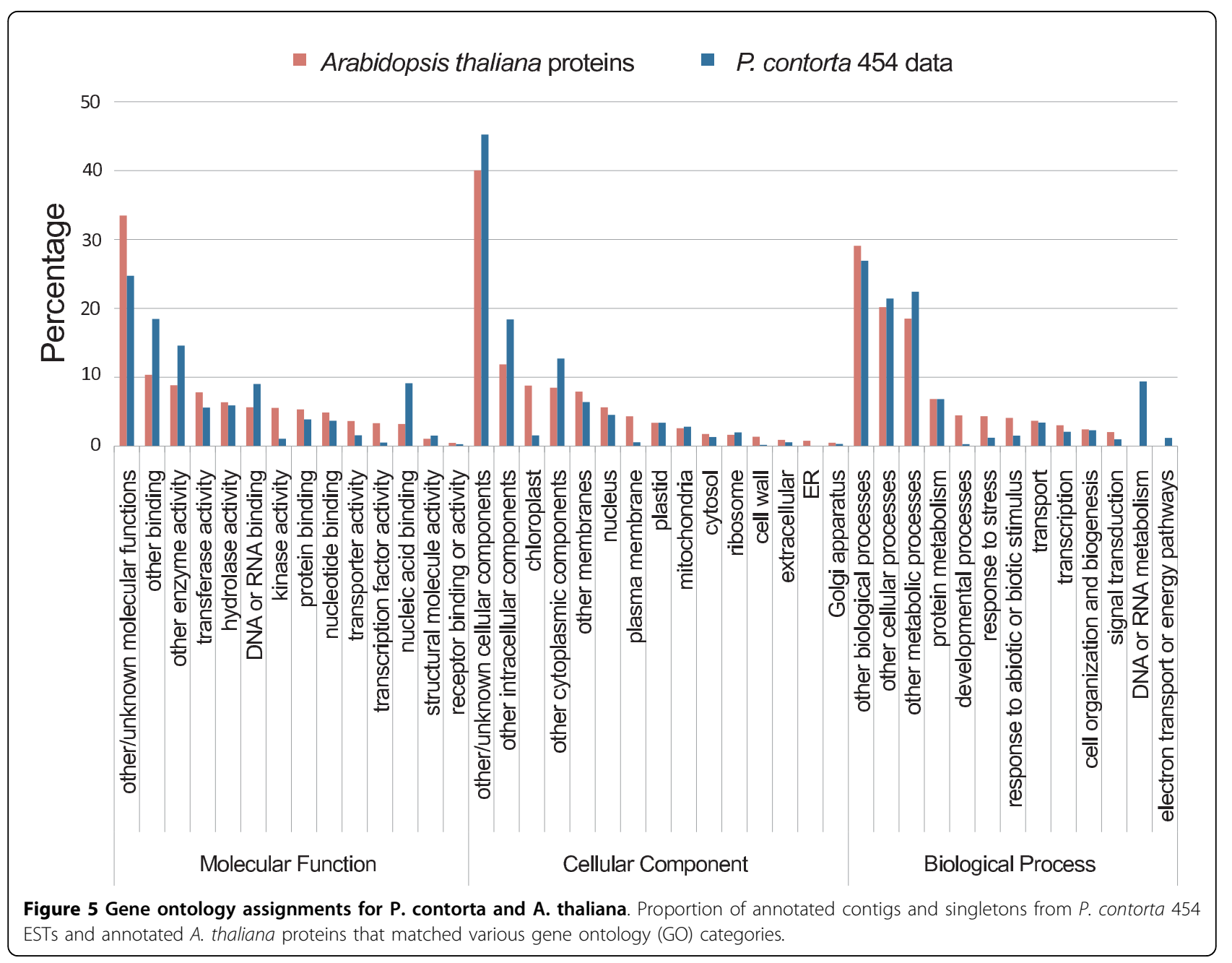




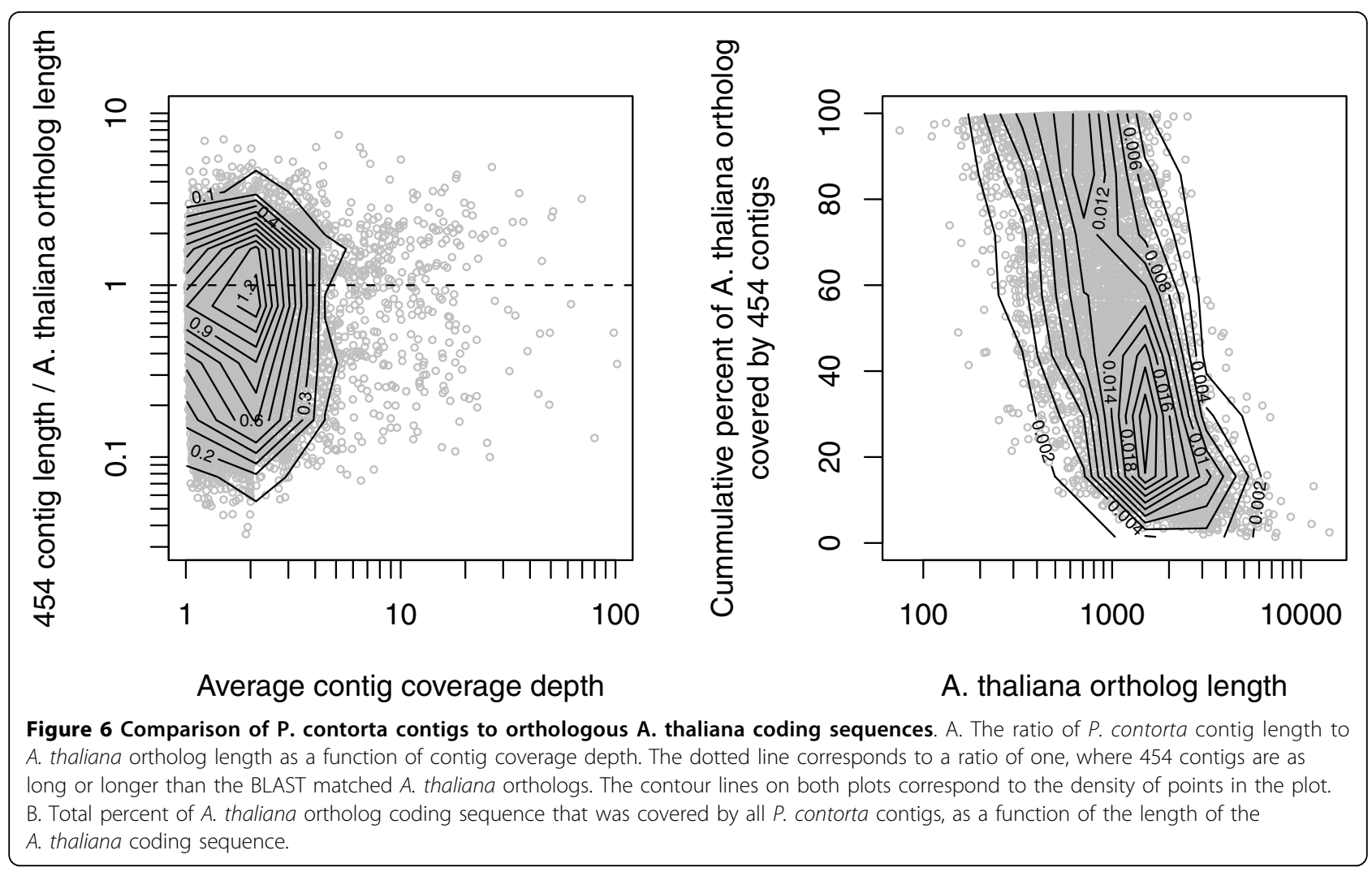

Table 4 Summary of plant ESTs with BLAST matches to retrotransposons

\begin{tabular}{lrrr}
\hline Organism & \# of ESTs & \# of ESTs matching RTs & \% Total \\
\hline Arabidopsis thaliana & $1,527,298$ & 920 & 0.0602 \\
Triticum aestivum & $1,066,854$ & 1,123 & 0.1053 \\
Vitis vinifera & 353,941 & 217 & 0.0613 \\
Populus nigra & 51,361 & 84 & 0.1635 \\
Populus tremula & 37,313 & 15 & 0.0402 \\
Populus trichocarpa & 89,943 & 94 & 0.1045 \\
Picea glauca & 297,913 & 210 & 0.0705 \\
Picea sitchensis & 168,675 & 54 & 0.0320 \\
Pinus pinaster & 27,847 & 15 & 0.0539 \\
Pinus taeda & 328,628 & 217 & 0.0660 \\
Pinus contorta & 586,372 & 22,862 & 3.8989 \\
\hline
\end{tabular}

Number of ESTs matching to 17 well-characterized plant retrotransposon sequences in BLASTn searches of large EST collections for 10 selected plant taxa and the entire set of $P$. contorta 454 reads.

name, known function, conserved motifs, or affiliated gene ontology terms. This suggests that the majority of the sequences that were similar to known retroelements are likely to reflect transcriptionally active retrotransposons, although a significant proportion (18\%) of retrotransposon-like sequences may represent transcribed regions containing short pieces of retroelement-like sequences.

\section{Marker identification and characterization}

Using Perl scripts we identified a total of 15,084 di-, tri-, or tetra-nucleotide SSR regions with a minimum of four contiguous repeating units in contigs from the referencebased and de novo assemblies (Table 5). We identified 1,841 potential SSRs in contigs aligned to the P. taeda unigenes in the reference-based assembly (Table 5), and a much larger number of SSRs in the contigs built through the de novo assembly. Di-nucleotide repeats were by far the most common SSRs in our ESTs, with tri- and tetra-nucleotide repeats being present at much smaller frequencies (Table 5). 7,906 SSRs occurred in contigs with BLAST matches to Uniref50 annotated proteins, of which a substantial number $(5,678)$ occurred in actual protein coding sequences. Although the majority of these contigs consisted of coding sequence, the density of SSRs was still higher in coding (0.0034 SSRs per bp) than non-coding regions (0.0018 SSRs per bp).

We were able to design high quality PCR primers with optimal expected product sizes and other properties that should facilitate successful amplification for a large portion these SSRs using BatchPrimer3 [42]. Primers were constructed for nearly a third of the SSRs identified, but quality priming sites occurred in a much higher proportion of tri- and tetra-nucleotide repeats than of dinucleotide repeats (Table 5). Information on the name 
Table 5 SSRs in 454 ESTs

\begin{tabular}{lrrr}
\hline Type of SSR repeat & Reference based assembly & De novo assembly of remaining reads & Total \\
\hline $\mathrm{Di}$ & $1,267(54)$ & $10,366(1,487)$ & $11,633(1,541)$ \\
Tri & $551(520)$ & $2,480(1,748)$ & $3,031(2,268)$ \\
Tetra & $23(19)$ & $397(192)$ & $420(211)$ \\
\hline Total & $1,841(593)$ & $13,243(3,427)$ & $15,084(4,020)$ \\
\hline
\end{tabular}

Numbers of di-, tri-, and tetranucleotide simple sequence repeats (SSRs) occurring in contigs from both the reference-based and de novo assemblies. In parentheses following the number of loci identified are the number of potentially amplifiable loci for which PCR primers were designed in BatchPrimer3 [42] with stringent criteria (see Additional file 1: Supplemental Table S1).

of the sequence containing each SSR, SSR motif, number of repeats, expected length of PCR product, sequences and positions of forward and reverse primers, and the GC content and melting temperature for each primer is included in Additional file 1: Supplemental Table S1. Of 96 loci tested for succesful PCR amplification in a single trial, $70 \%$ successfully amplified in $P$. contorta and $65 \%$ successfully amplified in P. ponderosa. In addition, more than $50 \%$ of the tested SSRs were polymorphic across panels of eight individuals of $P$. contorta and $P$. ponderosa.

Using Seqman Pro (DNASTAR, Inc.), we identified a substantial number of SNPs in our largest and most deeply covered contigs. The 20 BLAST-annotated contigs with the largest numbers of reads contained 301 SNPs with alternate alleles in at least $20 \%$ of reads and minimum coverage depth of $10 \times$. These contigs represent 27,496 bp of sequence data, giving a SNP occurrence rate of 1.1 SNPs per $100 \mathrm{bp}$. Across all the contigs with more than 20 reads (658 contigs containing 614,125 nucleotides) we identified a total of 3,707 (0.6 high quality SNPs per $100 \mathrm{bp}$ ) high quality SNPs with frequency of the alternative allele being at least $20 \%$ and a minimum coverage depth of $8 \times$.

\section{Discussion}

Normalized cDNA collections from multiple tissues and individuals allowed us to sequence a large fraction of the $P$. contorta transcriptome using 454 pyrosequencing. The approximately $1.8 \times 10^{8}$ base pairs of data produced here represent a substantial sequence resource for $P$. contorta and will contribute to genomic data available for the genus Pinus. Below we discuss the characteristics and level of transcriptome coverage in our data, the abundance of transcriptionally active retrotransposons, the characterization of different molecular marker types, and future prospects for population genomics studies.

\section{Assembly and annotation}

As with other recent studies $[5,7,15,16]$, our results indicate that short reads from 454 sequencing runs can be effectively assembled and used to readily characterize the gene space of non-model organisms. The large number and length of reads generated from a half plate pyrosequencing run on a 454 GS XLR70 Titanium instrument resulted in a relatively high expected average transcriptome coverage depth $(3.6 \times)$. As expected with the increased read lengths from the 454 GS XLR70 Titanium instrument, our contigs were on average larger $($ mean $=500 \mathrm{bp})$ than those assembled in previous studies that used earlier 454 technologies with shorter reads (e.g., 197 bp, [5]; 247 bp, [7]; 440 bp, [16]). In addition, a large percentage of our contigs were greater than $800 \mathrm{bp}$ in length and had good coverage depth (Figs. 3, 4). High quality of assembled contigs was indicated by a high proportion of contigs matching to known proteins using BLAST searches and by the ready PCR amplification of SSR markers developed in contigs.

Nonetheless, a relatively small portion (48\%) of reads were assembled into contigs, which is significantly less than that reported for several other recent 454 transcriptome assemblies (e.g., 91\%, [5]; 88\%, [7]; 90\%, [16]). As a consequence, coverage depth and contig length was lower than expected for many contigs. Large numbers of singletons could result from a variety of causes, including the assembly algorithm used, artifacts of cDNA normalization, genes expressed at low levels, contaminants from other organisms such as bacterial or fungal parasites, or 454 sequencing errors [12]. Transcriptome assembly is additionally hindered by alternative splicing, among other issues [5,17]. Long repeat regions, which are particularly common in pines, are known to cause difficulties with de novo assembly $[12,43]$. One factor potentially influencing difficulty in assembly is the abundance of both simple and complex repeats. Although we removed a substantial number of reads prior to assembly, both long SSR regions and retroelements were prevalent in our data and could have contributed to difficulty with assembly. Additional filtering steps could improve the fraction of reads assembling into contigs [44], although this could also involve an overall loss of information. Nonetheless, many singletons were high quality reads and matched to proteins in BLAST searches, highlighting that they are still a very important source of information. Given that next generation sequencing projects will soon become common in non-model organisms, increases in the amount and 
quality of data should result in the improvement of $d e$ novo assembly algorithms over time [17].

Estimating the number of genes and the level of transcript coverage represented in an EST collection is an important issue for transcriptome sequencing projects, but is difficult or impossible without a completely annotated reference genome sequence. We indirectly evaluated transcriptome coverage breadth by first determing the number of unique genes detected in our sequence collection using BLAST. The large number of sequences that matched in BLAST searches to unique proteins (Table 2) indicates that our 454 sequencing reads identified a substantial portion of the genes in P. contorta. A large portion of the 17,321 unique genes had best BLAST hits to plant proteins, with a smaller portion hitting to other taxonomic groups (Table 3). A similarly large number of these were assigned to a wide range of gene ontology categories (Fig. 5), indicating that a wide diversity of transcripts are represented by our sequence data. Furthermore, many of the contigs and singletons without BLAST hits likely represent additional genes not represented in the annotated protein databases we searched, or genes that lack BLAST matches due to short length. If we assume a similar number of genes occur in pines as in Arabidopsis (25,000, [31]), our annotated sequences are likely to represent more than half of the genes in P. contorta. To evaluate the extent to which individual transcripts were fully covered, we quantified coverage of full length $A$. thaliana orthologs by our 454 contigs. A substantial number of $A$. thaliana orthologs were entirely covered by 454 contigs, with increasingly complete coverage for contigs with higher coverage depths (Fig. 6A). Similarly, the completeness of ortholog coverage decreased with decreasing contig coverage depth and increasing length of the $A$. thaliana sequence, indicating that additional sequencing would be warranted for more comprehensive transcriptome coverage (Fig. 6A, B).

ESTs with BLAST hits to distant taxa may represent proteins not well-characterized in closely related taxa, but could also represent RNA from other organisms present in sampled tissues. The substantial number of BLAST hits to known fungal proteins in our ESTs (Table 3) is most likely due to the presence of fungi in the needles and or developing conelets we sampled. This is not surprising given the prevalence of endophytic and symbiotic fungi in conifer tissues [45,46]. Similarly, [5] reported matches in BLAST searches of their 454 ESTs to a substantial number of non-metazoan proteins, and identified the presence of an important intracellular parasite known to affect butterfly population dynamics. Such findings point to the utility of largescale transcriptome sequencing and annotation for detecting parasites or symbionts present in sampled in tissues, just as is done more generally in environmental genomics [47].

\section{Transcriptionally active retrotransposons}

Retrotransposons are known to play a prominent role in the evolution of genome size and complexity across eukaryotes [48]. For example $30-50 \%$ of the mammalian genome is composed of retroelements [9], and they comprise a substantial proportion of most plant genomes $[49,50]$. In addition, the proliferation of retrotransposons may be a major factor contributing to the large genome sizes of pines, where retrotransposons may account for almost $2 \%$ of the genome [34]. Although they are typically not actively transcribed into RNA, their presence in EST collections indicates that they can be transcriptionally active, and in some cases at very high levels (Table 4; [51,52]). BLAST searches of known retrotransposon sequences in plants as well as results from BLAST annotation of our contigs and singletons indicate that more than $6 \%$ of our 454 reads represent retrotransposon-like sequences. They are present in our data at a level an order of magnitude higher than in comparable EST collections for other plants, and two orders of magnitude higher than in other conifers (Table 4). We recognize that a variety of issues may contribute to this result, including possible variability of cDNA normalization across studies or tissues. Nonetheless this result highlights the abundance of retrotransposons that may occur in large-scale EST sequencing projects and deserves attention for several reasons. First, repetitive DNA is a known difficulty for assembly of short sequences into contigs, and the prevalence of retrotransposon sequences could contribute to assembly difficulties. Consequently, the characterization of retrotransposons in our 454 ESTs will be useful for planning future transcriptome sequencing in this species, where it may be desirable to use laboratory methods to remove retroelements from the cDNA sequencing pool and enrich for non-repetitive templates. Finally, given that a major goal of our study was molecular marker development, identification of the retrotransposon-derived reads is crucial to avoid developing and genotyping molecular markers residing in such sequences.

\section{Marker identification and characterization}

Polymorphic genetic markers are important for research involving population genetic structuring, demography, relatedness, and the genetic basis of adaptive traits [53-55]. Next generation transcriptome sequencing leads to superior resources for the development of such markers not only because of the enormous amount of sequence data in which markers can be identified, but also because discovered markers are gene-based. Such markers are advantageous because they facilitate the 
detection of functional variation and the signature of selection in genomic scans or association genetic studies $[2,56]$. Currently few genetic marker resources exist for $P$. contorta (but see [57]). The large number of SSRs and SNPs we detected provide a wealth of markers potentially useful to applications ranging from population genetics, linkage mapping, and comparative genomics, to gene-based association studies aimed at understanding the genetic control of adaptive traits.

EST-based SSRs are advantageous compared to SSRs located in non-transcribed regions owing to their higher amplification rates and cross-species transferability [58]. We have identified large numbers of SSRs in our 454 EST collection, and were able to design high quality PCR primers for nearly a third of these regions (Table 5). Moreover, a large number of these SSRs occur in the protein coding sequences of annotated contigs representing genes of known or predicted identity and function. That most of these SSRs occur in coding sequences $(5,678$ out of 7,906$)$ and not UTRs is perhaps surprising. Another possibly surprising result is that the majority of SSRs in the coding regions were di-nucleotide repeats $(78.4 \% \mathrm{di}-, 19.4 \%$ tri-, and $2.1 \%$ tetranucleotide repeats). These di-nucleotide SSRs were typically short $(<20 \mathrm{bp})$, and coding regions with di-nucleotide repeats almost always contained more than one SSR, although these were not always in close proximity where they could be considered interrupted repeats.

We were also able to design primers for a large subset of the SSRs located in regions where $P$. contorta ESTs were aligned to P. taeda ESTs (Table 5). As priming sites should be highly conserved, these markers are likely to be transferable to related species, and may represent a valuable source of genetic markers for the genus Pinus. We were able to successfully amplify a large percentage of polymorphic SSR loci in initial tests with $P$. contorta and $P$. ponderosa, validating the quality of our assembled contigs and the utility of the SSRs produced. Our ongoing work is assessing amplification rates, polymorphism levels and cross-species transferability for hundreds of these SSRs across multiple species of pines. The density of SSRs detected in 454 sequencing projects depends on numerous factors including the template sequenced and the criteria used in recognizing SSRs, and has varied greatly across recent studies $[5,59,60]$. Nonetheless, these studies and our own highlight the value of 454 sequencing as a cost and time effective route for rapid SSR discovery.

Because of the deep and redundant coverage produced over many genes, pyrosequencing of cDNA is ideal for SNP discovery and characterization $[5,6,16]$. Although our sampling was limited to four different individuals, high quality SNPs are abundant in our contigs. In addition, most of these SNPs reside in annotated genes, which will allow the identification of reading frame and facilitate more detailed analyses on the significance of molecular variation. The SNP frequency in our contigs $(0.6 / 100 \mathrm{bp})$ is in the general range of that reported in other studies using 454 pyrosequencing of cDNA pooled from multiple individuals (e.g., [6] 0.33/100 bp [5] 0.67/ $100 \mathrm{bp}$, [16] 0.49/100 bp, [61] 0.72/100 bp). Studies such as these highlight the value of next generation sequencing projects for SNP discovery and characterization. Similar to studies on P. taeda [62,63] and Picea glauca [8], the availability of large numbers of SNPs should facilitate population genomic and gene-based association studies in P. contorta.

\section{Conclusions}

Pines are among the most ecologically and economically important plant species on Earth. Yet, the size and complexity of their genomes has hindered the development of genomic resources for many taxa in the genus. The 303,480 unique sequences in this 454 EST collection represent a major genomic level resource for $P$. contorta, and will be useful for comparative genomic studies in pines. This highlights the utility of high-throughput transcriptome sequencing as a fast and cost-effective road to rapidly obtain information on coding genetic variation in pines. Because of large and minimally structured populations, high levels of nucleotide diversity, and rapid decay of linkage disequilibrium, conifers represent excellent subjects for association genetic studies $[27,64]$. Nonetheless, the enormous size of the pine genome means that millions of markers will be required for the fine-scale mapping of traits. Consequently, utilizing SNPs occurring in candidate genes or in ESTs is a promising avenue for association genetics in pines $[27,64]$. Until recently, association genetic studies for adaptive variation have been limited in conifers (but see $[62,65])$ due to the high cost and time required by traditional routes for the development of marker resources. The ability to investigate the genetic basis of adaptive traits in these trees should increase as groups working on pine genomic resources continue to build large EST data sets through next generation sequencing approaches. The thousands of SNP and SSR markers in our 454 ESTs should enable population genomic and gene-based association studies $[53,66]$. Such analyses should contribute to understanding patterns of adaptive variation across the genome and to identifying the genetic basis of adaptive traits.

\section{Methods}

\section{Sequencing and assembly}

Fresh needles and developing conelets were sampled from four individual $P$. contorta trees in the Medicine Bow National Forest of south central Wyoming (USA). 
RNA extraction, cDNA synthesis, and 454 sequencing were performed by staff at the biotechnology company GATC (GATC, Inc.). Total RNA was isolated using a CTAB-based protocol and was further purified with the NucleoSpin RNA XS kit (Macherey and Nagel, Inc.). RNA was pooled from the four individuals for cDNA synthesis. From this pool, poly(A)+ RNA was prepared, and first-strand cDNA synthesis was primed with an N6 randomized primer. 454 adapters A and B (Roche Life Sciences, Inc.) were ligated to the 5 ' and 3 ' ends of the cDNA, and the cDNA was PCR-amplified using a proof reading enzyme. One cycle of denaturation and reassociation of the cDNA was used to obtain N1 cDNA. Single stranded cDNA was used for hybridization rather than double stranded cDNA, and normalization of the cDNA template was achieved by separating reassociated double stranded cDNA from the single stranded cDNA by passing the mixture over a hydroxylapatite column $[15,67]$. Following hydroxylapatite chromotography, single stranded cDNA was PCR amplified. One $\mu \mathrm{g}$ of cDNA was sequenced in two half-plate runs on a 454 GS XLR70 Titanium genomic sequencer (Roche, Inc.). Although technical difficulties with the first run resulted in a smaller than expected number of reads, these reads were included in analyses as they were still of high quality. Files containing the sequences and quality scores have been deposited at NCBIs Short Read Archive (accession SRA012089). 454 primer sequences were trimmed from all reads prior to assembly. Because repetitive DNA is a known problem for the assembly of short pyrosequencing reads, and repeats are abundant in conifer genomes, we removed a large number of reads from the full data set to avoid sequences that could interfere with assembly. We discarded reads with simple sequence repeats longer than $50 \mathrm{bp}$, and a substantial number of reads matching to three well-characterized conifer retrotransposon sequences (see below). We also removed reads with average quality scores less than 18. We used Seqman Ngen (DNAstar, Inc.) to assemble reads into contigs, as this program has been successful in assembling 454 sequences from transcriptomes $[5,68,69]$ and other programs created to assemble next generation sequencing data often assemble few EST reads into contigs $[14,17]$. The assembly algorithm employed by Ngen is an extension of work by [70] that allows the incorporation of quality scores for individual nucleotides, and can be specifically parameterized for assembling short pyrosequencing reads. Sequences are placed into a hash table of overlapping subsequences, before sequential assembly of overlapping reads occurs with near constant memory usage (for a detailed explanation of the algorithm see [71]).
22To explore the effects of parameter settings on the outcome of assemblies, we ran de novo assemblies with a range of minimum match percentages (85\%, $90 \%$ and $95 \%)$, match lengths $(19,23$, and $25 \mathrm{bp})$, and gap penalties (30 and 50) (Table 1). Analyses presented in this paper are based on a combination and reference-based and de novo assemblies. We first executed a referencebased assembly using a set of 18,921 unigenes constructed from $P$. taeda Sanger ESTs http://www.ncbi. nlm.nih.gov/UniGene/UGOrg.cgi?TAXID=3352. For this assembly, we used a minimum match size of 19 nucleotides, match percentage of $88 \%$, mismatch penalty of 18 , and gap penalty of 30 . We used a lower minimum match percentage here because of substantial divergence between taxa. We utilized this approach not only to facilitate assembly but also to produce a set of contigs containing sequences conserved between $P$. contorta and P. taeda. These served as a basis for characterizing molecular markers likely to have high cross-species transferability. Reads that did not join into contigs in the reference-based assembly were entered into a de novo assembly with a more stringent minimum match percentage. This assembly was run with a minimum match size of 19 nucleotides, match percentage of $93 \%$, mismatch penalty of 18 , and gap penalty of 30 . The resulting contigs and remaining singletons were then combined into a single set (Fig. 2).

\section{Annotation}

To assess the coverage and quality of our assembly, we first used local BLASTx [35] to align both contigs and singletons to the UniRef50 15.4 [36] and the TAIR9 Arabidopsis thaliana [37] annotated protein databases using an $E$ value threshold of $10^{-6}$. BLASTx results were passed through a custom Perl pipeline that summarized information and produced tab-delimited tables with accession numbers, gene name, taxonomic ID, query length, ortholog sequence length, sequence alignment, $E$ value, and bit score for each protein accession with matches in BLAST searches. To estimate the proportion of annotated contigs and singletons that matched to unique genes in the two databases, these files were then filtered for redundancy in protein accessions. Assignment of gene ontology (GO) terms to ESTs with BLASTx matches was then performed by importing the accession numbers for the BLASTx hits to unique proteins into Blast2go (version 2.3.6; http://www.blast2go. org/). Blast2go is an automated tool for the assignment of gene ontology terms to BLAST hits and was designed for use with novel sequence data [39]. We also generated gene ontology assignments for A. thaliana annotated proteins to compare the distribution of functional 
annotations in $P$. contorta to that from a plant with a well-characterized transcriptome.

\section{Assessment of retrotransposon abundance}

Because preliminary analyses identified a very large number of retrotransposon like sequences in our data, we quantified the presence of retroelements in the raw 454 data and the set of contigs and singletons with BLASTn searches using two approaches. First, we used BLASTn to search for 17 known plant retrotransposon sequences in our entire set of 454 reads and in large EST collections from other selected plant taxa. These retrotransposon sequences represent complete sequences from the NCBI Nucleotide database http:// www.ncbi.nlm.nih.gov/entrez?db=nuccore that match queries of 'copia-like' and 'gypsy-like' in plant taxa. Additionally, complete retrotransposon sequences from conifer genomes were selected, including IFG7, PpRT1, and Gymny [32-34]. Ten species were chosen for comparison of retrotransposon abundance on the basis of taxonomic relatedness to our study species and the number of ESTs available. Entire EST collections available in dbEST for these species, as well as our complete set of $P$. contorta 454 reads, were compared to the 17 retrotransposon sequences using BLASTn with an $E$ value threshold of $10^{-6}$. Second, following BLAST annotation of contigs and singletons, we further searched for sequences representing proteins associated with retroelements by identifying sequences that had the terms "copia", "gag", "pol", "retroelement", "integrase", "reverse transcriptase" and "retrotransposon" in their annotation.

\section{Marker identification and characterization}

We wrote Perl programs to identify SSRs in our contigs and to identify a subset of these that reside in contigs where reads from $P$. contorta were assembled onto $P$. taeda unigenes. We located di-, tri-, and tetra-nucleotide SSRs with lengths less than 50 bp and with a minimum of 4 contiguous repeating units, which provided a large number of candidate SSRs. We determined which SSRs occurred in coding sequences of genes by extracting the aligned portions of sequences having BLAST matches to annotated protein coding orthologs, and then using the same algorithm as above to detect SSRs in both the aligned and remaining portions of these contigs. The design of high quality PCR primers is crucial for the development of molecular markers that can be readily usable, and suitable priming sites will not exist for all of the loci we identified. To determine the loci that represent good candidates for PCR amplification, we used the program BatchPrimer3 [42] to construct PCR primers in the flanking regions of SSRs. This program identifies SSRs, allows the control of many parameters to facilitate high quality primer construction, and can simultaneously process thousands of sequences. We designed primers in the flanking regions of SSRs that were a minimum of $12 \mathrm{bp}$ long and used stringent criteria to design genetic markers of desired PCR product length and with a high probability of amplification. We created primers with a minimum GC content of $30 \%$, with a melting temperature between 52 and $62^{\circ} \mathrm{C}$ and a maximum $4^{\circ} \mathrm{C}$ difference between primers, and positioned primers to obtain PCR products between 100 and $450 \mathrm{bp}$ long. We also constrained primer construction so that the end of each primer contained a GC clamp (the last two nucleotides were $\mathrm{G}$ or $\mathrm{C}$ ). Additional settings used for primer design are available from the authors upon request.

We tested 96 of these primer sets for succesful SSR amplification in several individuals in $P$. contorta and $P$. ponderosa. PCR reactions consisted of 50-100 ng total genomic DNA; 2 pmol of each primer; $0.5 \mathrm{mM}$ each of dATP, dCTP, dGTP, and dTTP; $1 \times$ PCR buffer; and 0.4 units of taq polymerase. All polymerase chain reaction amplifications were performed with the following conditions; $94^{\circ} \mathrm{C}$ for five minutes, followed by 32 cycles of $94^{\circ}$ $\mathrm{C}$ for one minute, $50^{\circ} \mathrm{C}$ for one minute, and $72^{\circ} \mathrm{C}$ for one minute, followed by a final extension step of $72^{\circ} \mathrm{C}$ for three minutes. We ran $12 \mu \mathrm{l}$ of each product out on $1.5 \%$ agarose gels stained with ethidium bromide, and scored each individual for successful amplification. A subset of SSR loci that amplified successfully were tagged with flourescently labelled M13 tails, run out on an ABI 3130 genetic analyzer (ABI, Inc.), and genotyped for polymorphism.

We also identified an abundance of SNPs in large contigs with high coverage depths using the SNP reporter feature in Seqman Pro (DNAstar, Inc.). We considered only SNPs with nucleotide variation, and disregarded indels. First, we enumerated and visually inspected high quality SNPs with coverage depth of at least $10 \times$ and with an alternate allele in a minimum of $20 \%$ of the reads for the 20 contigs containing the largest number of reads. Second, we assessed the presence of high quality SNPs in all contigs containing greater than 25 reads. Here we counted SNPs at sites where coverage depth was at least $8 \times$, and where alternate alleles were present at a minimum frequency of $20 \%$.

Additional file 1: Supplementary File S1 - Primer sequences for SSR loci. Information for SSR primers designed in BatchPrimer3 using stringent criteria. Provided for each locus is information on the sequence ID, location of SSR, location of both primers, primer sequences, length of primer sequences, melting temps, GC\%, SSR motif, motif length, SSR sequence, and SSR length. 


\section{Acknowledgements}

We appreciate constructive comments on earlier versions of the manuscript from Zach Gompert and three anonymous reviewers. An NSF DBI award (0701757) to C. A. Buerkle and an NSF DEB award (0344503) to C. W. Benkman supported this work. K. Geist was supported by an NIH INBRE program at the University of Wyoming for training undergraduates in bioinformatics. We thank DNAstar for technical guidance and expertise and for graciously allowing use of their Segman NGen and Lasergene software packages.

\section{Author details}

'Department of Botany, University of Wyoming, Laramie, WY 82071, USA. ${ }^{2}$ Department of Biology, Beloit College, Beloit, WI 53511, USA. ${ }^{3}$ Department of Molecular Biology, Laramie, WY 82071, USA. ${ }^{4}$ Department of Zoology and Physiology, University of Wyoming, Laramie, WY 82071, USA.

\section{Authors' contributions}

TLP organized and planned the research, contributed to all aspects of analysis, and drafted the manuscript. KSG wrote Perl scripts, contributed to analyses involving BLAST and retrotransposon sequences and contributed to manuscript preparation. JAG provided programming expertise, wrote bioinformatics scripts, and guided analyses involving BLAST and SSR characterization. CWB contributed to conceptual planning of the research, provided funds, and contributed to manuscript preparation. CAB provided funding, computational guidance, and was substantially involved in research design, data analysis, and manuscript preparation. All authors have read and approved the final manuscript

Received: 20 January 2010 Accepted: 16 March 2010 Published: 16 March 2010

\section{References}

1. Stinchcombe JR, Hoekstra HE: Combining population genomics and quantitative genetics: finding the genes underlying ecologically important traits. Heredity 2007, 100:158-170.

2. Bouck A, Vision T: The molecular ecologist's guide to expressed sequence tags. Molecular Ecology 2007, 16(5):907-924.

3. Andersen JR, Lubberstedt T: Functional markers in plants. Trends in Plant Science 2003, 8(11):554-560.

4. Emrich SJ, Barbazuk WB, Li L, Schnable PS: Gene discovery and annotation using LCM-454 transcriptome sequencing. Genome Research 2007, 17:69-73.

5. Vera JC, Wheat CW, Fescemyer HW, Frilander MJ, Crawford DL, Hanski I, Marden JH: Rapid transcriptome characterization for a nonmodel organism using 454 pyrosequencing. Molecular Ecology 2008, 17(7):1636-1647.

6. Barbazuk WB, Emrich SJ, Chen HD, Li L, Schnable PS: SNP discovery via 454 transcriptome sequencing. Plant Journal 2007, 51(5):910-918.

7. Novaes E, Drost DR, Farmerie WG, Pappas GJ Jr, Grattapaglia D, Sederoff RR, Kirst M: High-throughput gene and SNP discovery in Eucalyptus grandis, an uncharacterized genome. BMC Genomics 2008, 9:312.

8. Namroud MC, Beaulieu J, Juge N, Laroche J, Bousquet J: Scanning the genome for gene single nucleotide polymorphisms involved in adaptive population differentiation in white spruce. Molecular Ecology 2008, 17(16):3599-3613.

9. Margulies M, Egholm M, Altman WE, Attiya S, Bader JS, Bemben LA, Berka J, Braverman MS, Chen YJ, Chen ZT, Dewell SB, Du L, Fierro JM, Gomes XV, Godwin BC, He W, Helgesen S, Ho CH, Irzyk GP, Jando SC, Alenquer MLI, Jarvie TP, Jirage KB, Kim JB, Knight JR, Lanza JR, Leamon JH, Lefkowitz SM, Lei M, Li J, Lohman KL, Lu H, Makhijani VB, McDade KE, McKenna MP, Myers EW, Nickerson E, Nobile JR, Plant R, Puc BP, Ronan MT, Roth GT, Sarkis GJ, Simons JF, Simpson JW, Srinivasan M, Tartaro KR, Tomasz A, Vogt KA, Volkmer GA, Wang SH, Wang Y, Weiner MP, Yu PG, Begley RF, Rothberg JM: Genome sequencing in microfabricated high-density picolitre reactors. Nature 2005, 437(7057):376-380.

10. Ellegren H: Sequencing goes 454 and takes large-scale genomics into the wild. Molecular Ecology 2008, 17(7):1629-1631.

11. Hudson ME: Sequencing breakthroughs for genomic ecology and evolutionary biology. Molecular Ecology Resources 2008, 8:3-17.
12. Pop M, Salzberg SL: Bioinformatics challenges of new sequencing technology. Trends in Genetics 2008, 24(3):142-149.

13. Wicker T, Schlagenhauf E, Graner A, Close TJ, Keller B, Stein N: 454 sequencing put to the test using the complex genome of barley. BMC Genomics 2006, 7:275.

14. Weber APM, Weber KL, Carr K, Wilkerson C, Ohlrogge JB: Sampling the Arabidopsis transcriptome with massively parallel pyrosequencing. Plant Physiology 2007, 144:32-42.

15. Kristiansson E, Asker N, Forlin L, Larsson DGJ: Characterization of the Zoarces viviparus liver transcriptome using massively parallel pyrosequencing. BMC Genomics 2009, 10:345.

16. Meyer E, Aglyamova GV, Wang S, Buchanan-Carter J, Abrego D, Colbourne JK, Willis BL, Matz MV: Sequencing and de novo analysis of a coral larval transcriptome using 454 GSFIx. BMC Genomics 2009, 10:219.

17. Wheat CW: Rapidly developing functional genomics in ecological model systems via 454 transcriptome sequencing. Genetica 2008.

18. Burns RM, Honkala BH: Silvics of North America, Conifers. Agriculture Handbook 654. Tech rep USDA, Washington DC 1990, 1.

19. Critchfield WB: Genetics of lodgepole pine. United States Forest Service Research Paper WO-37 1980.

20. Smith CC: Coevolution of pine squirrels (Tamiasciurus) and conifers. Ecological Monographs 1970, 40(3):349-371.

21. Benkman CW, Holimon WC, Smith JW: The influence of a competitor on the geographic mosaic of coevolution between crossbills and lodgepole pine. Evolution 2001, 55(2):282-294.

22. Benkman CW, Parchman TL, Favis A, Siepielski AM: Reciprocal selection causes a coevolutionary arms race between crossbills and lodgepole pine. American Naturalist 2003, 162(2):182-194.

23. Benkman CW, Siepielski AM: A keystone selective agent? Pine squirrels and the frequency of serotiny in lodgepole pine. Ecology 2004, 85(8):2082-2087.

24. Lotan JE: The role of cone serotiny in lodgepole pine forests. Symposium Proceedings Washington State University, Pullman, Washington, USABaumgartner DM 1975, 471-495.

25. Arno SF: Forest fire history in the Northern Rockies. Journal of Forestry 1980, 78(8):460-465

26. Hicke JA, Jenkins JC: Mapping lodgepole pine stand structure susceptibility to mountain pine beetle attack across the western United States. Forest Ecology and Management 2008, 255(5-6):1536-1547.

27. Neale DB, Savolainen O: Association genetics of complex traits in conifers. Trends in Plant Science 2004, 9(7):325-330.

28. Guevara MA, Soto A, Collada C, Plomion C, Savolainen O, Neale DB, González-Martínez SC, Cervera MT: Genomics applied to the study of adaptation in pine species. Invest Agrar: Sist Recur For 2005, 14(3):292-306.

29. Neale DB: Genomics to tree breeding and forest health. Current Opinion in Genetics \& Development 2007, 17(6):539-544.

30. Krutovsky KV, Neale DB: Nucleotide diversity and linkage disequilibrium in cold-hardiness- and wood quality-related candidate genes in Douglas-fir. Genetics 2005, 171(4):2029-2041.

31. Bevan M, Walsh S: The Arabidopsis genome: A foundation for plant research. Genome Research 2005, 15(12):1632-1642.

32. Kossack DS, Kinlaw CS: IFG, a gypsy-like retrotransposon in Pinus (Pinaceae), has an extensive history in pines. Plant Molecular Biology 1999, 39(3):417-426.

33. Rocheta M, Cordeiro J, Oliveira M, Miguel C: PpRT1: the first complete gypsy-like retrotransposon isolated in Pinus pinaster. Planta 2007, 225(3):551-562.

34. Morse AM, Peterson DG, Islam-Faridi MN, Smith KE, Magbanua Z, Garcia SA, Kubisiak TL, Amerson HV, Carlson JE, Nelson CD, Davis JM: Evolution of genome size and complexity in Pinus. PLOS ONE 2009, 4(2):e4332.

35. Altschul S, Madden T, Schaffer A, Zhang JH, Zhang Z, Miller W, Lipman D: Gapped BLAST and PSI-BLAST: A new generation of protein database search programs. Nucleic Acids Research 1997, 25(17):3389-3402.

36. Suzek BE, Huang H, McGarvey P, Mazumder R, Wu CH: UniRef: comprehensive and non-redundant UniProt reference clusters. Bioinformatics 2007, 23(10):1282-1288.

37. Swarbreck D, Wilks C, Lamesch P, Berardini TZ, Garcia-Hernandez M, Foerster H, Li D, Meyer T, Muller R, Ploetz L, Radenbaugh A, Singh S, Swing V, Tissier C, Zhang P, Huala E: The Arabidopsis Information 
Resource (TAIR): gene structure and function annotation. Nucleic Acids Research 2008, 36(Sp. Iss. SI):D1009-D1014.

38. Beldade P, Rudd S, Gruber JD, Long AD: A wing expressed sequence tag resource for Bicyclus anynana butterflies, an evo-devo model. BMC Genomics 2006, 7:130.

39. Conesa A, Gotz S, Garcia-Gomez JM, Terol J, Talon M, Robles M: Blast2GO: a universal tool for annotation, visualization and analysis in functional genomics research. Bioinformatics 2005, 21(18):3674-3676.

40. Lomax J: Get ready to GO! A biologist's guide to the Gene Ontology. Briefing in Bioinformatics 2005, 6(3):298-304.

41. DeMarco R, Kowaltowski AT, Machado AA, Soares MB, Gargioni C, Kawano T, Rodrigues V, Madeira AMBN, Wilson RA, Menck CFM, Setubal JC, Dias-Neto E, Leite LCC, Verjovski-Almeida S: Saci-1, -2 , and -3 and Perere, four novel retrotransposons with high transcriptional activities from the human parasite Schistosoma mansoni. Journal of Virology 2004, 78(6):2967-2978

42. You FM, Huo N, Gu YQ, Luo Mc, Ma Y, Hane D, Lazo GR, Dvorak J, Anderson OD: BatchPrimer3: A high throughput web application for PCR and sequencing primer design. BMC Bioinformatics 2008, 9:253.

43. Holt RA, Jones SJM: The new paradigm of flow cell sequencing. Genome Research 2008, 18(6):839-846.

44. Huse SM, Huber JA, Morrison HG, Sogin ML, Mark Welch D: Accuracy and quality of massively parallel DNA pyrosequencing. Genome Biology 2007, 8(7):R143.

45. Hata K, Futai K, Tsuda M: Seasonal and needle age-dependent changes of the endophytic mycobiota in Pinus thunbergii and Pinus densiflora needles. Canadian Journal of Botany 1998, 76(2):245-250.

46. Ganley RJ, Brunsfeld SJ, Newcombe G: A community of unknown, endophytic fungi in western white pine. Proceedings of the National Academy of Sciences 2004, 101(27):10107-10112.

47. Venter JC, Remington K, Heidelberg JF, Halpern AL, Rusch D, Eisen JA, Wu DY, Paulsen I, Nelson K, Nelson W, Fouts D, Levy S, Knap AH, Lomas MW, Nealson K, White O, Peterson J, Hoffman J, Parsons R, BadenTillson $\mathrm{H}$, Pfannkoch C, Rogers YH, Smith HO: Environmental genome shotgun sequencing of the Sargasso Sea. Science 2004, 304(5667):66-74.

48. Charlesworth B, Sniegowski P, Stephan W: The evolutionary dynamics of repetitive DNA In eukaryotes. Nature 1994, 371(6494):215-220.

49. Kidwell MG: Transposable elements and the evolution of genome size in eukaryotes. Genetica 2002, 115:49-63.

50. Lisch D: Epigenetic regulation of transposable elements in plants. Annual Review of Plant Biology 2009, 60:43-66.

51. Vicient $C$, Jaaskelainen $M$, Kalendar $R$, Schulman A: Active retrotransposons are a common feature of grass genomes. Plant Physiology 2001 125(3):1283-1292.

52. Faulkner GJ, Kimura Y, Daub CO, Wani S, Plessy C, Irvine KM, Schroder K, Cloonan N, Steptoe AL, Lassmann T, Waki K, Hornig N, Arakawa T, Takahashi H, Kawai J, Forrest ARR, Suzuki H, Hayashizaki Y, Hume DA, Orlando V, Grimmond SM, Carninci P: The regulated retrotransposon transcriptome of mammalian cells. Nature Genetics 2009, 41(5):563-571.

53. Luikart G, England PR, Tallmon D, Jordan S, Taberlet P: The power and promise of population genomics: from genotyping to genome typing. Nature Reviews Genetics 2003, 4:981-994.

54. Avise JC: Molecular Markers, Natural History, and Evolution London, UK: Chapman and Hall 2004.

55. Beaumont MA: Adaptation and speciation: what can $F_{\mathrm{ST}}$ tell us? Trends in Ecology \& Evolution 2005, 20:435-440.

56. Vasemagi A, Nilsson J, Primmer CR: Expressed sequence tag-linked microsatellites as a source of gene-associated polymorphisms for detecting signatures of divergent selection in Atlantic salmon (Salmo salar L.). Molecular Biology and Evolution 2005, 22(4):1067-1076.

57. Liewlaksaneeyanawin C, Ritland CE, El-Kassaby YA, Ritland K: Single-copy, species-transferable microsatellite markers developed from loblolly pine ESTs. Theoretical and Applied Genetics 2004, 109(2):361-369.

58. Barbara T, Palma-Silva C, Paggi GM, Bered F, Fay MF, Lexer C: Cross-species transfer of nuclear microsatellite markers: potential and limitations. Molecular Ecology 2007, 16(18):3759-3767.

59. Castoe TA, Poole AW, Gu W, de Koning APJ, Danza JM, Smith EM, Pollock DD: Rapid identification of thousands of copperhead snake (Agkistrodon contortrix) microsatellite loci from modest amounts of 454 shotgun genome sequence. Molecular Ecology Resources 2010, 10(2):341-347.
60. Tangphatsornruang S, Somta P, Uthaipaisanwong P, Chanprasert J, Sangsrakru D, Seehalak W, Sommanas W, Tragoonrung S, Srinives P: Characterization of microsatellites and gene contents from genome shotgun sequences of mungbean (Vigna radiata (L.) Wilczek). BMC Plant Biology 2009, 9:137.

61. Hahn DA, Ragland GJ, Shoemaker DD, Denlinger DL: Gene discovery using massively parallel pyrosequencing to develop ESTs for the flesh fly Sarcophaga crassipalpis. BMC Genomics 2009, 10:234.

62. González-Martínez SC, Wheeler NC, Ersoz E, Nelson CD, Neale DB: Association Genetics in Pinus taeda L. I. Wood Property Traits. Genetics 2007, 175:399-409

63. Eckert AJ, Pande B, Ersoz ES, Wright MH, Rashbrook VK, Nicolet CM, Neale DB: High-throughput genotyping and mapping of single nucleotide polymorphisms in loblolly pine (Pinus taeda L.). Tree Genetics \& Genomes 2009, 5:225-234.

64. Gonzalez-Martinez SC, Krutovsky KV, Neale DB: Forest-tree population genomics and adaptive evolution. New Phytologist 2006, 170(2):227-238.

65. Eckert AJ, Wegrzyn JL, Pande B, Jermstad KD, Lee JM, Liechty JD, Tearse BR, Krutovsky KV, Neale DB: Multilocus patterns of nucleotide diversity and divergence reveal positive selection at candidate genes related to cold hardiness in coastal Douglas Fir (Pseudotsuga menziesii var. menziesii). Genetics 2009, 183:289-98.

66. Storz JF: Using genome scans of DNA polymorphism to infer adaptive population divergence. Molecular Ecology 2005, 14:671-688.

67. Soares MB, Bonaldo MD, Jelene P, Su L, Lawton L, Efstratiadis A: Construction and characterization of a normalized CDNA library. Proceedings of the National Academy of Sciences of the United States of America 1994, 91(20):9228-9232.

68. Guerrero FD, Down SE, Djikeng A, Wiley G, Macmil S, Saldivar L, Najar F, Roe BA: A Database of Expressed Genes From Cochliomyia hominivorax (Diptera: Calliphoridae). Journal of Medical Entomology 2009, 46(5):1109-1116

69. Mattanovich D, Graf A, Stadlmann J, Dragosits M, Redl A, Maurer M, Kleinheinz M, Sauer M, Altmann F, Gasser B: Genome, secretome and glucose transport highlight unique features of the protein production host Pichia pastoris. Microbial Cell Factories 2009, 8:29.

70. Allex CF, Baldwin SF, Shavlik JW, Blattner FR: Increasing consensus accuracy in DNA fragment assemblies by incorporating flourescent trace representations. Proceedings International Conference on Intelligent Systems for Molecular Biology 1997.

71. Durfee T, Nelson R, Baldwin S, Plunkett G III, Burland V, Mau B, Petrosino JF, Qin X, Muzny DM, Ayele M, Gibbs RA, Csoergo B, Posfai G, Weinstock GM, Blattner FR: The complete genome sequence of Escherichia coli DH10B: Insights into the biology of a laboratory workhorse. Journal of Bacteriology 2008, 190(7):2597-2606.

\section{doi:10.1186/1471-2164-11-180}

Cite this article as: Parchman et al:: Transcriptome sequencing in an ecologically important tree species: assembly, annotation, and marker discovery. BMC Genomics 2010 11:180.

\section{Submit your next manuscript to BioMed Central and take full advantage of:}

- Convenient online submission

- Thorough peer review

- No space constraints or color figure charges

- Immediate publication on acceptance

- Inclusion in PubMed, CAS, Scopus and Google Scholar

- Research which is freely available for redistribution

Submit your manuscript at www.biomedcentral.com/submit
C Biomed Central 